Cross-modal orienting of visual attention

Running head: Cross-modal orienting of visual attention

\title{
Cross-modal Orienting of Visual Attention
}

\author{
Steven A. Hillyard ${ }^{1}$, Viola S. Störmer ${ }^{2}$, Wenfeng Feng ${ }^{3}$, \\ Antigona Martinez ${ }^{1,4}$, John J. McDonald ${ }^{5}$ \\ ${ }^{1}$ Department of Neuroscience, University of California at San Diego, USA \\ 2Department of Psychology, Harvard University, Cambridge, USA \\ ${ }^{3}$ Department of Psychology, SooChow University, China \\ ${ }^{4}$ Nathan Kline Institute for Psychiatric Research, USA \\ ${ }^{5}$ Department of Psychology, Simon Fraser University, Canada
}

\begin{abstract}
Corresponding author:
Steven A. Hillyard, University of California at San Diego, USA

shillyard@ucsd.edu
\end{abstract}

Keywords: cross-modal attention, ERPs, alpha rhythm, multisensory processing

(C) 2015. This manuscript version is made available under the Elsevier user license http://www.elsevier.com/open-access/userlicense/1.0/ 


\begin{abstract}
This article reviews a series of experiments that combined behavioral and electrophysiological recording techniques to explore the hypothesis that salient sounds attract attention automatically and facilitate the processing of visual stimuli at the sound's location. This cross-modal capture of visual attention was found to occur even when the attracting sound was irrelevant to the ongoing task and was non-predictive of subsequent events. A slow positive component in the event-related potential (ERP) that was localized to the visual cortex was found to be closely coupled with the orienting of visual attention to a sound's location. This neural sign of visual cortex activation was predictive of enhanced perceptual processing and was paralleled by a desynchronization (blocking) of the ongoing occipital alpha rhythm. Further research is needed to determine the nature of the relationship between the slow positive ERP evoked by the sound and the alpha desynchronization and to understand how these electrophysiological processes contribute to improved visual-perceptual processing.
\end{abstract}




\section{Visual Stimuli Influence Auditory Perception, and Vice Versa}

The auditory and visual systems interact in many ways, perhaps most strikingly in the ventriloquist illusion (a visual event biases the perceived location of a concurrent sound) and the McGurk effect (visual image of speaker's mouth alters perception of speech sounds) (Calvert et al., 2004; Murray \& Wallace, 2012). Conversely, auditory events can produce dramatic alterations in visual perception, such as when two sounds surround a single flash and produce the percept of two flashes (Shams et al., 2000). Insights into the neural bases of these well-known cross-modal interactions have emerged from electrophysiological and neuroimaging studies. Vision-induced modulations of neural activity in auditory cortical regions have been demonstrated for the ventriloquist (Bonath et al., 2007) and McGurk (Baart et al., 2014; Ganesh et al., 2014) illusions, and auditory-induced activity in the visual cortex has been associated with the Shams doubleflash illusion (Mishra et al., 2007).

It is well-documented that selectively attending to the location of a stimulus in one modality not only facilitates the processing of that stimulus but also of stimuli of other modalities that occur at the attended location (Spence \& Driver 2004; McDonald et al., 2012). This cross-modal property of spatial attention was demonstrated early on in electrophysiological studies in which auditory and visual stimuli were presented in random order at left and right locations, and observers attended to one modality at one location at a time; it was found that event-related brain potentials to all stimuli at the attended location were enhanced, both for the relevant and (to a lesser extent) the irrelevant modality (Hillyard et al., 1984; Eimer and Schröger, 1998; Teder-Sälejärvi et al., 1999). Similarly, behavioral studies have shown that when attention is cued to a 
specific location in space the observer's response to a subsequent target is facilitated regardless of the modalities of the cue and target stimuli (Driver \& Spence, 2004). Such facilitation was observed both for endogenous cueing (observers voluntarily direct attention to the location symbolically indicated by the cue) and exogenous (involuntary) cueing (the cue is salient and attracts attention, but its location is not predictive of target location). The present article reviews a series of studies carried out over the past 15 years that explore the behavioral and neural mechanisms by which a non-predictive auditory stimulus facilitates the processing of a subsequent visual event at the same location. By making non-invasive recordings of ERPs and EEG during task performance, our recent studies have identified auditory-evoked patterns of neural activity in the visual cortex that are closely coupled with the cross-modal facilitation of visual processing.

\section{Salient Sounds Capture Visual Attention}

Several lines of research converge on the hypothesis that a salient sound naturally attracts attention to its location and facilitates the processing of a subsequent visual event at the same location (McDonald et al. 2012; 2013a). The basic design of these studies presents an initial sound (the cue) from a free-field speaker situated to the left or right of the midline, which is followed after a short interval (from one to several hundreds of milliseconds) by a visual stimulus (the target) that occurs either at the same location as the sound or at a mirror-image location in the opposite visual field (or in some studies, at both locations simultaneously). Critically, the left-right position of the cue is nonpredictive of the location(s) of the subsequent target event(s), so that any locationspecific influence of the cue on target processing must be purely involuntary. 
In our first study of this type McDonald et al. (2000) used a signal detection paradigm in which the auditory cue (brief noise burst) was followed after an SOA (stimulus-onset asynchrony) of 100-300 ms by a flashed array of LEDs presented at the same or opposite-field location on an unpredictable basis. The task was to report whether or not a faint, threshold-level target flash preceded the brighter LED array, which served both as a mask and as a post-cue that defined the location to be reported. It was found that the perceptual sensitivity (d') for detecting the faint target was greater when the target + mask array was presented at the same location as the preceding auditory cue than when the array appeared in the opposite visual field. Similar effects of auditory cues on visual target detection were reported by Dufour (1999) and Frassinetti et al. (2002). McDonald and colleagues proposed that the lateralized auditory cue attracted attention to its location involuntarily, which resulted in enhanced perceptual processing of a subsequent visual target at the sound's location. ERP recordings showed that this crossmodal facilitation of visual processing was associated with an increased neural response in the ventral extrastriate visual cortex elicited by the target/mask complex, which may be mediated by a feedback projection from the polymodal region of the superior temporal lobe (McDonald et al., 2003).

A subsequent experiment investigated whether the cross-modal cueing of attention by an auditory cue would speed up the perceived onset time of a co-localized visual event (McDonald et al., 2005). Previous studies had used time-order judgment (TOJ) tasks to demonstrate that when attended and unattended visual stimuli are presented simultaneously, the attended stimulus appears to onset earlier in time (Shimojo et al., 1997; Shore et al., 2001). Using a TOJ paradigm, McDonald et al. (2005) 
presented observers with a lateralized auditory cue followed by a pair of brief flashes, one at the location of the sound and the other in the opposite visual field. The time offset between the two flashes was varied unpredictably over a small range from trial to trial, and the observer was charged with reporting which flash appeared to occur first. The prior auditory cue produced a dramatic effect, such that the flash on the cued side was judged to occur earlier on the majority of trials (including $79 \%$ of the simultaneous-flash trials). Only when the flash on the uncued side preceded the cued-side flash by around $70 \mathrm{~ms}$ were the two flashes judged to be simultaneous.

To study the neural basis of this strong cross-modal cueing effect, McDonald et al. (2005) examined ERPs elicited by the paired flashes on trials when they were actually presented simultaneously. The paired flashes elicited an early positivity (starting at 90$100 \mathrm{~ms}$ ) that was enlarged over the visual cortex contralateral to the side of the auditory cue, but there were no differences in the timing of the peaks of the visual ERP that corresponded to the $70 \mathrm{~ms}$ shift in perceived timing. This suggested that the apparent temporal precedence of the flash on the cued side was due to an enlarged neural response in the contralateral visual cortex rather than to a speeding of neural transmission in the visual pathways. Moreover, the finding of an enhanced visual cortex ERP associated with the speeded perception of the flash on the cued side provided evidence that this TOJ effect was truly an effect of the cue on visual perception rather than a biasing of the postperceptual decision in favor of the cued side (Schneider \& Bavelier, 2003; Santangelo \& Spence, 2008).

Another striking example of how the cross-modal cueing of attention influences the timing of visual perception can be seen in the "illusory line motion" (ILM) effect 
(Hikosaka et al., 1996). When a horizontal line is briefly flashed following a cue to attend to one end of the line, the observer typically reports that the line grows from the cued end to the uncued end, even though the entire line was flashed simultaneously. The ILM illusion appears to be closely related to the aforementioned TOJ effect, since the observer's perceptual report is that the cued end of the line is seen first. McDonald and colleagues (2013a) investigated the neural basis of the ILM illusion in a situation where a brief sound preceded a horizontal line flashed on a video screen with a variable SOA of 100-300 ms; the sound was presented unpredictably from a speaker that was situated either near the right or the left end of the line. A psychophysical analysis using graded line growths in either direction showed that the observers judged a fully stationary line to grow from the cued end on $76 \%$ of the trials. Concurrent ERP recordings showed that this ILM illusion, like the TOJ effect, was associated with an enlarged positive component elicited by the line that was localized to ventral visual cortex contralateral to the auditory cue and began about $90-100 \mathrm{~ms}$ after the onset of the line. This visual cortex modulation provided further evidence that the influence of the auditory cue on visual timing judgments is a sensory-perceptual effect rather than a consequence of postperceptual response bias.

\section{Auditory Cues Alter Visual Appearance}

A long-debated question in psychology is whether directing attention to a visual stimulus can alter its subjective appearance, for example by making it appear brighter or higher in contrast than when unattended (James, 1890). Carrasco et al. (2004) obtained an affirmative answer to this question in an experiment where attention was cued by 
briefly flashing a black dot at a left or right location on a video screen, and this cue was followed immediately by a pair of Gabor patches, one at the cued location and the other in the opposite visual field. On trials when the two patches actually had the same physical contrast, observers judged the patch at the pre-cued location as being higher in contrast. Carrasco et al.'s conclusion that attention can alter appearance was quickly challenged by proponents of alternative explanations; chief among these were proposals that the apparent increase in contrast may have been due to purely sensory interactions between the visual cue and the visual target (Schneider \& Komlos, 2008) or to a decisionlevel response bias in favor of the cued location (Prinzmetal et al., 2008).

In light of the aforementioned evidence that salient sounds can capture visual attention, Störmer et al. (2009) investigated whether an auditory cue might also modulate the perceived luminance contrast of a subsequent visual target. In a cross-modal version of Carrasco et al.'s experiment, an auditory cue (noise burst) was presented unpredictably at a right or left location, followed immediately (SOA of $150 \mathrm{~ms}$ ) by a bilateral pair of Gabor patches that varied in their relative contrasts (Figure 1A). On trials where the patches were physically identical, it was found that the patch at the auditorily cued location was judged to be higher in contrast than the patch in the opposite visual field (55\% vs 45\%) (Figure 1B). Concurrent ERP recordings showed that this cueing effect on luminance contrast was associated with an enlarged occipital positivity that began around 90-100 ms after the Gabor patches and reached a maximum at 120-140 ms; this enhanced positivity was localized to a source in the ventral extrastriate visual cortex contralateral to the cued side (Figure $1 \mathrm{C}, \mathrm{D})$. The amplitude of this contralateral 


\section{Cross-modal orienting of visual attention}

positivity was systematically correlated with the probability of reporting the cued-side target as being higher in contrast (Figure 1E).

\section{A ERP to the visual targets}

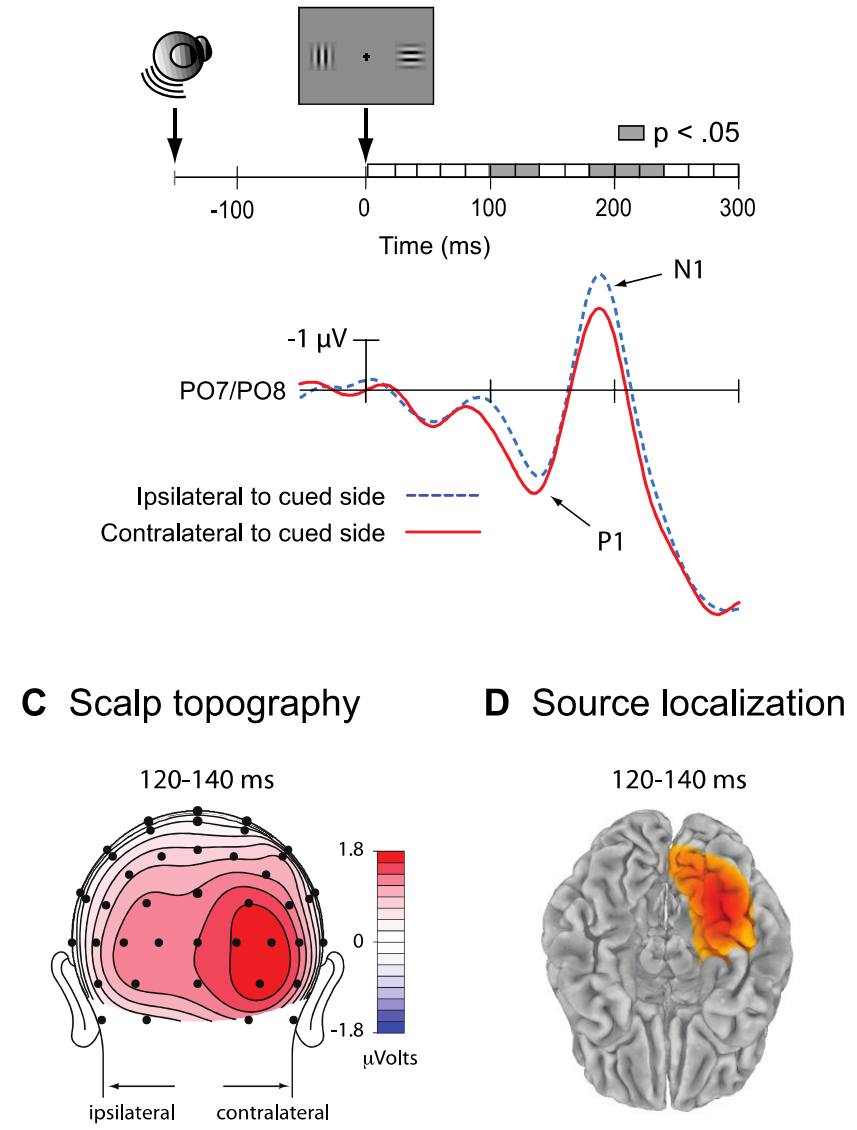

B Psychometric function

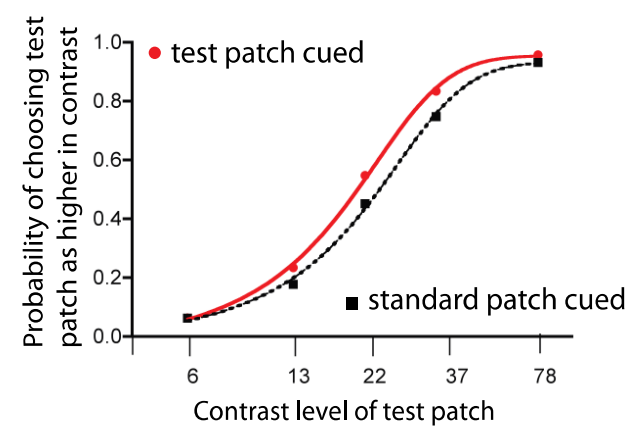

E Neural-behavioral correlation

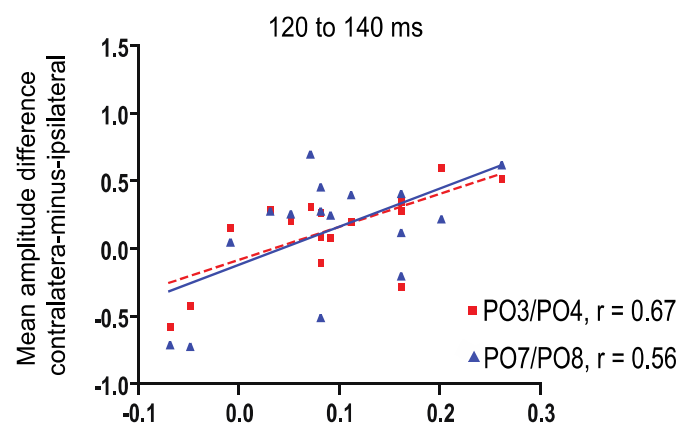

Difference of the probability of choosing cued target as higher contrast minus the probability of choosing uncued target as higher in contrast

Figure 1. Results from contrast-appearance experiment of Störmer et al. (2009). A. Stimulus sequence and grand-average ERP elicited by bilateral, equal-contrast Gabor patches. On each trial a lateralized auditory cue was presented $150 \mathrm{~ms}$ before a bilateral pair of Gabor patches that varied in their relative contrast. On equal-contrast trials observers judged the patch on the side of the auditory cue to be higher in luminance contrast on the majority of trials, and this behavioral effect was associated with a larger ERP 
positivity over the hemisphere contralateral to the cue. B. Psychophysical results showing that the cued patch had a higher probability of being judged as higher in contrast. C. Scalp topography map showing larger positivity over the occipital scalp contralateral to the auditorily cued side in the ERP elicited by the equal-contrast Gabor patches. The voltage is mapped over the time interval 120-140 ms after onset of the patches. D. Source localization estimated by the LAURA algorithm of the contralateral positivity shown in C. Maximal current density was located in the ventral occipito-temporal cortex contralateral to the sound cue. E. Correlation between the amplitude of the contralateral positive ERP (over 120-140 ms) and an individual's tendency to report the cued-side target to be higher in contrast. Observers with larger lateralized responses in the visual cortex showed a higher probability of reporting the patch on the cued side as being higher in contrast.

Störmer et al. (2009) proposed that the sound had provoked an involuntary crossmodal orienting of visual-spatial attention to its location, and this capture of attention resulted in the higher perceived contrast of the Gabor patch at the cued location. The use of an auditory cue ruled out the possibility of unimodal sensory interactions between cue and target, and the ERP modulations in visual cortex at 90-140 ms provided strong evidence that the cross-modal cueing of attention affects visual appearance at an early sensory-perceptual level rather than by influencing later decision or response processes. ERP studies over the past several decades have demonstrated that neural activity patterns associated with stimulus relevance, classification, and decision-making are not elicited earlier than 150 ms after stimulus onset (Luck \& Kappenman, 2011).

It is interesting to note that this enlarged contralateral positivity at $90-140 \mathrm{~ms}$ that is elicited by bilateral visual stimuli when preceded by a unilateral auditory cue (McDonald et al. 2005, 2013a; Störmer et al. 2009) strongly resembles the enhanced "P1" positivity that has been observed in unimodal visual attention experiments (reviewed in Hopfinger et al. 2004). For example, Heinze et al. (1994) presented bilateral pairs of visual symbols to subjects who were cued endogenously to attend to the symbols in either the left or the right visual field. Under these conditions the bilateral stimuli elicited an early positivity (the P1 at 90-150 ms) that was enlarged over the occipital scalp 


\section{Cross-modal orienting of visual attention}

contralateral to the attended visual field. Source localization confirmed by positron emission tomography estimated the neural generators of this P1 asymmetry to lie in the ventral extrastriate visual cortex. These similarities in timing and source localization of the early positivity suggest that the attentional modulation of visual processing by an exogenous auditory cue may utilize a common mechanism with the endogenous cueing of visual attention.

\section{Neural Bases of Involuntary Cross-modal Cueing}

As described above, there is ample evidence that spatially non-predictive sounds can affect visual perception, but little is known about the underlying neural mechanisms. In particular, one might expect to find that salient sounds would activate neurons in the visual cortex, either via direct connections from auditory to visual cortex or mediated through multisensory convergence zones (Driver \& Noesselt, 2008). To investigate whether spatially non-predictive sounds would elicit activity in the visual cortex, McDonald et al. (2013b) recorded ERPs to lateralized sounds under several different task conditions, including conditions where the sounds were irrelevant and where no visual stimuli were presented at all. The specific experimental conditions tested were the following: (1) the lateralized sound preceded a bilateral pair of Gabor patches - the paradigm of Störmer et al. (2009); (2) the lateralized sound preceded a pair of target tone bursts; (3) the lateralized sounds were completely irrelevant and were presented in a randomized sequence with paired bilateral target tones; and (4) the lateralized sounds were completely irrelevant and were presented in a randomized sequence with central target tones. 
In all of these task conditions the lateralized sounds elicited a slow positive potential shift over the interval $200-500 \mathrm{~ms}$ that was larger over the posterior scalp contralateral to the sound and was localized to the ventral extrastriate occipital cortex (Figure 2, A, B, C). This Auditory-evoked Contralateral Occipital Positivity (ACOP) was elicited even in the conditions where only auditory stimuli were presented and where the lateralized sounds were neither task-relevant nor predictive of the randomly occurring target tones. These results suggest that even irrelevant sounds can activate the visual cortex with some degree of automaticity. A likely interpretation of this cross-modal influence would be that a salient sound automatically (involuntarily) attracts spatial attention to its location and that spatial attention is inherently linked to visual processing. It remains to be seen, however, whether this cross-modal activation of visual cortex is completely automatic — that is, inevitably present under all task conditions — or whether it can be modified by some manipulations of attention.

A Sound-elicited ERP

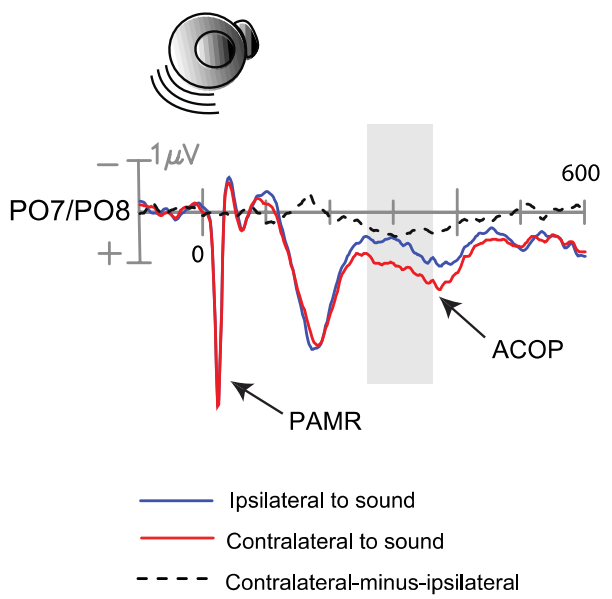

B ACOP scalp topography

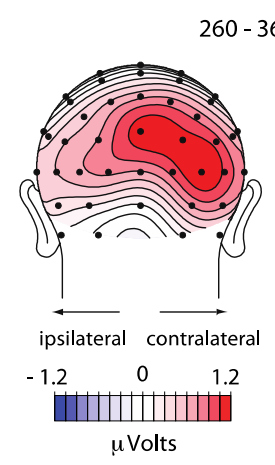

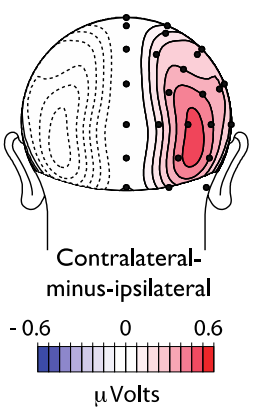

D Neural-behavioral correlation

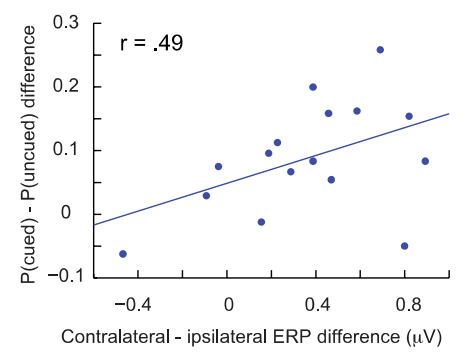


Figure 2. ERP elicited by the lateralized (non-predictive) auditory cue (noise bursts) in the paradigm of Störmer et al (2009) (see Figure 1). A. ERP shown was elicited on sound-only trials where the Gabor patches were omitted to show the auditory-evoked ERP without contamination by the visual ERP to the patches. ERP waveforms are collapsed over scalp sites contralateral and ipsilateral to the side of the sound. Shaded area shows the interval (260-360 ms) over which the lateralized ACOP component was quantified as the contralateral minus ipsilateral positivity. B. Voltage topography of the ACOP shows larger positivity over the contralateral occipital scalp (right side of head). C. Cortical source of the ACOP was estimated by dipole modeling and current density mapping to lie in the ventral occipital cortex. D. Between-subject correlation between the amplitude of the ACOP and the individuals' tendency to judge the Gabor patch on the cued side as being higher in contrast than the patch on the uncued side. Data from McDonald et al. (2013b).

Evidence that the ACOP is indeed conducive to enhanced visual processing was obtained by McDonald et al. (2013b) in the condition where the lateralized sound was followed by a pair of Gabor patches. It was found that observers who displayed the largest ACOP amplitudes (contralateral minus ipsilateral positivity in the visual cortex) also had the strongest tendency to judge the Gabor patch contralateral to the sound as having higher luminance contrast than the ipsilateral patch (Figure 2D). This suggests that the ACOP reflects neural activity in the visual cortex that enhances the sensory strength of visual inputs from the location to which the auditory cue captured attention.

A further linkage between the ACOP and visual perceptual processing was demonstrated by Feng et al. (2014). In this study the lateralized sound preceded a unilateral masked target letter ( $\mathrm{T}$ or $\mathrm{L}$ ) that was presented unpredictably at either the same location as the sound (valid trials) or in the opposite visual field (invalid trials) (Figure 3A). The observer's task was to make a forced-choice T vs. L discrimination. The SOA between the spatially non-predictive sound and the visual target was extended to $400 \mathrm{~ms}$ so that the ACOP could be measured on a trial by trial basis in the interval 
preceding the target. It was found that the ACOP amplitude was strongly predictive of visual discrimination accuracy. On validly cued trials the ACOP was large (and highly significant) when the letter discrimination was correct (valid-correct trials) but was near zero when the discrimination was incorrect (valid-incorrect trials) (Figure 3B).

Interestingly, on invalidly cued trials the ACOP was of intermediate amplitude and did not differ between trials with correct versus incorrect discriminations (Figure 4A). As in previous studies, the ACOP was localized by source modeling to the ventral-lateral extrastriate visual cortex (Figure 4B).

The finding of intermediate ACOP amplitudes on invalid trials can be explained if we assume that the ACOP is associated with an attentional process that facilitates visual processing at the cued location but does not affect processing at the opposite location. The trial to trial distribution of ACOP amplitudes (calculated as the contralateral minus ipsilateral difference) must be the same for valid and invalid trials, since with nonpredictive cueing the subject cannot know in advance whether the visual target will occur on the cued or uncued side. Therefore, on validly cued trials with a high ACOP the target discrimination accuracy will be higher than on validly cued trials with a low ACOP, but on invalidly cued trials the trial to trial variations in ACOP amplitude will not affect discrimination performance and the mean ACOP amplitude will be an intermediate average across the entire trial to trial range. 
A Experimental design

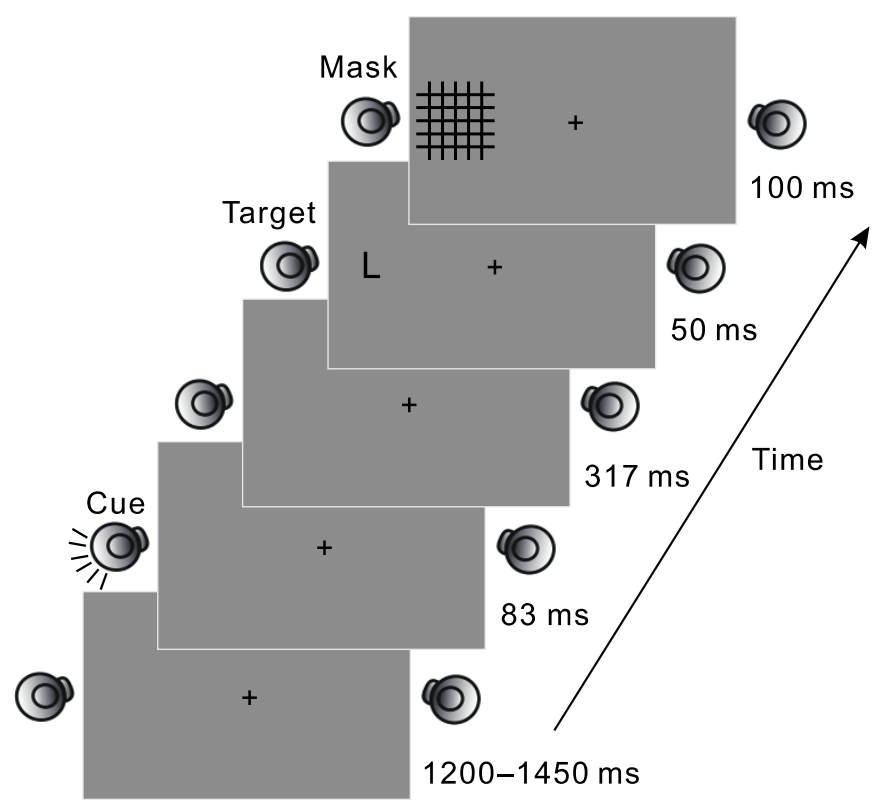

B ACOP for valid correct trials

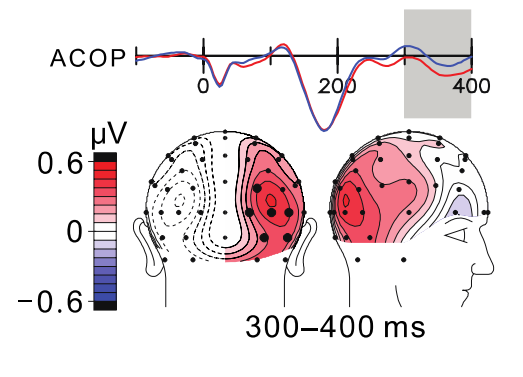

ACOP for valid incorrect trials

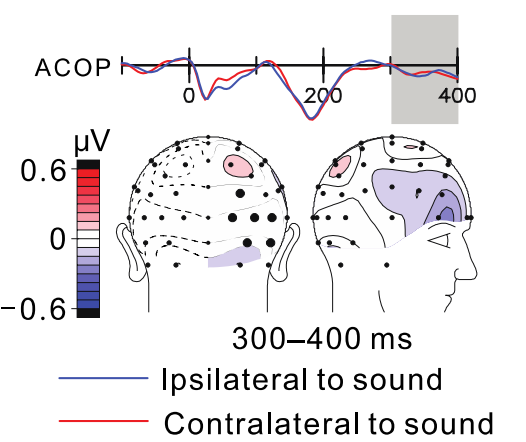

Figure 3. ACOP recorded in the study of Feng et al. (2014). A. Schematic of the experimental design. On each trial a lateralized sound was followed after an SOA of $400 \mathrm{~ms}$ by a target letter ( $\mathrm{L}$ in this example) and masking grid. Side of sound was not predictive of side of target. $\mathbf{B}$. ACOP waveforms and scalp topographies on valid trials (target appeared on same side as sound). ACOP (in shaded area) was much larger on trials when the T vs. L discrimination was correct than when incorrect.

These findings of Feng et al. (2014) further support the hypothesis that the ACOP represents lateralized neural activity in the visual cortex engendered by the involuntary orienting of visual attention to the non-predictive sound's location, and that this crossmodal activation improves the discriminative processing of the subsequent visual target at that location. The surprising finding of no difference in ACOP amplitude between correct and incorrect discriminations of invalidly cued targets can again be accounted for by assuming that the attentional processes associated with ACOP improve the processing of targets at the validly cued location, but do not suppress the processing of invalidly 
cued targets. If invalid targets were suppressed we would expect to find a larger ACOP (more positive in the hemisphere contralateral to the auditory cue) preceding incorrect than correct discriminations of invalidly cued targets.

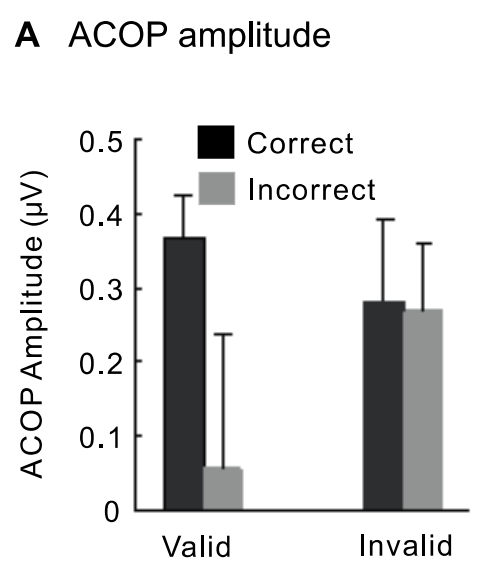

B ACOP source localization

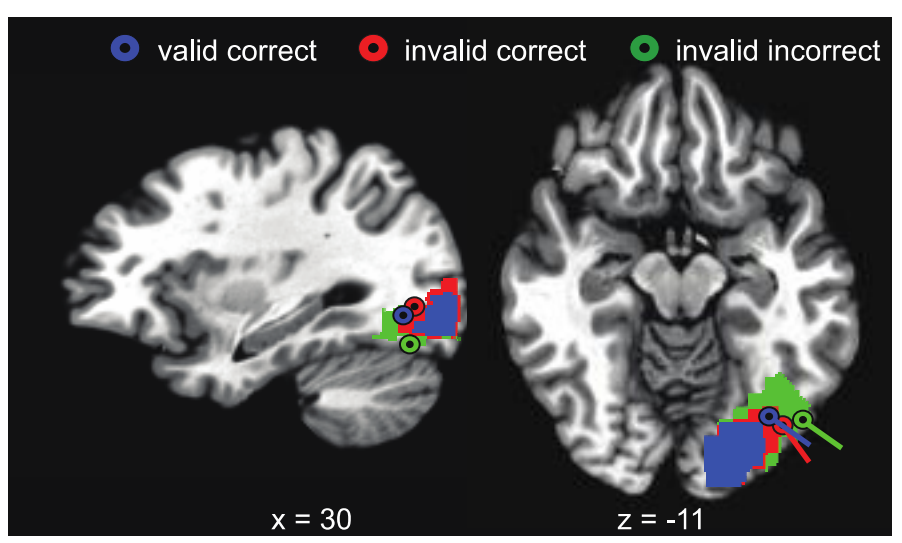

Figure 4. A. ACOP amplitude (contralateral minus ipsilateral positivity over the interval 300-400 $\mathrm{ms}$ ) was much larger for correct than incorrect trials when targets were validly cued but not when targets were invalidly cued. B. Estimated sources of the ACOP under different cueing conditions were all localized to the ventral occipital cortex as determined by both dipole modeling and current density mapping with LAURA.

In several ways, the ACOP resembles another lateralized ERP component called the Late Directing Attention Positivity (LDAP), which has been associated with voluntary shifts of attention (Harter et al., 1989; Hopf \& Mangun, 2000). Whereas the ACOP is triggered by salient, peripheral sounds that presumably capture attention reflexively, the LDAP is observed after the appearance of a centrally presented symbolic cue that indicates where an impending visual target is likely to appear. As its name implies, the LDAP emerges later than the ACOP (400-500 ms after cue onset). Although it has been hypothesized to reflect some aspects of target preparation in visual cortex (Hopf \& 
Mangun, 2000), the LDAP is time-locked to the cue rather than to the subsequent target (Van Velzen et al. 2002; Green \& McDonald, 2010). Like the ACOP, the LDAP is maximal over the posterior scalp and can even be observed in the absence of taskrelevant visual targets (Green, Teder-Sälejärvi, \& McDonald, 2005; Störmer, Green, \& McDonald, 2009). These characteristics suggest that the ACOP may be analogous to an "early LDAP" that is triggered reflexively by salient sounds. The two components may not reflect identical processes, however. In particular, the LDAP appears to reflect increased activity in the ipsilateral hemifield rather than the contralateral hemifield (McDonald \& Green, 2008). Thus, the ACOP might reflect enhanced visual processing in the cued hemifield, while the LDAP might reflect suppression of visual processing in the to-be-ignored hemifield.

\section{Involuntary Cross-Modal Orienting of Attention Modulates the Alpha Rhythm}

It is well known that the voluntary orienting of visual attention is accompanied by modulations of the occipital alpha rhythm $(8-14 \mathrm{~Hz})$ of the ongoing EEG (for reviews see Foxe \& Snyder, 2011; Hanslmayr et al., 2011; Thut et al., 2012). In particular, when attention is directed voluntarily to one side of the visual field (e.g., by a symbolic cue), the alpha band power typically decreases over the hemisphere contralateral to the attended field while reportedly increasing over the ipsilateral hemisphere (Green \& McDonald, 2010; Worden et al., 2000; Thut et al., 2006; Kelly et al., 2006; Handel et al., 2011; Capilla et al., 2014; Marshall et al., 2015). In many of these studies the degree of alpha reduction (i.e., "blocking" or "desynchronization" of the alpha rhythm) was correlated with improved perceptual processing of visual events at the attended location 
(e.g., Ergenoglu et al., 2004; Thut et al., 2006; Hanslmayr et al., 2007; Van Dijk et al., 2008; Mazaheri et al., 2014). Hemispherically lateralized modulations of the alpha rhythm have been interpreted in terms of a facilitation of visual processing of attended inputs (associated with a decrease in contralateral alpha amplitude) and/or an active inhibition of inputs from non-attended regions of the visual field (associated with an increase in ipsilateral alpha amplitude) (Green \& McDonald, 2010; Jensen \& Mazaheri, 2010; Foxe \& Snyder 2011; Klimesch, 2012; Payne et al., 2013).

In light of this evidence that the voluntary allocation of attention is associated with modulations of the occipital alpha rhythm, Störmer et al. (submitted) investigated whether the involuntary orienting of attention to a lateralized sound might also provoke a modulation of the alpha rhythm in the visual cortex. Of particular interest was to examine the possible relationship between alpha blocking and the ACOP that is elicited by salient auditory stimuli and was found to be associated with enhanced visual processing (Feng et al. 2014). Störmer et al. (submitted) re-analyzed the EEG data obtained in the purely auditory experiments reported by McDonald et al. (2013b) in the frequency domain using wavelets, and alpha power changes were compared with the concurrently recorded ACOP. As shown in Figure 5, the ACOP elicited by lateralized task-irrelevant sounds presented in a randomized sequence with central target tones (Experiment 4 of McDonald et al., 2013b) was paralleled by a lateralized alpha blocking that was greater in the hemisphere contralateral to the sound. The time course of the alpha blocking was similar to that of the ACOP, with onsets at around $200 \mathrm{~ms}$ and maximal amplitudes over 300-500 ms after the sound. Moreover, the ACOP (contralateral minus ipsilateral positivity) and the lateralized alpha blocking (contralateral 
minus ipsilateral alpha decrement relative to prestimulus baseline) had nearly identical scalp distributions, with maximal amplitudes over the ventral-lateral occipital scalp. It should be noted that the alpha amplitude was reduced (blocked) in both hemispheres following the irrelevant, non-predictive sounds, with a greater reduction in the hemisphere contralateral to the sound (Figure 5C). This contrasts with reports that alpha was increased in the hemisphere ipsilateral to the attended visual field when attention was directed voluntarily (Green \& McDonald, 2010; Jensen 2010; Foxe \& Snyder, 2011; Klimesch, 2012; Payne et al. 2013). This difference in alpha blocking patterns suggests that there may be a fundamental difference between the mechanisms of voluntary and involuntary cueing of spatial attention. Whereas the voluntary allocation of spatial attention involves both a facilitation of processing in the attended visual field (associated with a contralateral decrease in alpha amplitude) and a suppression of processing in the opposite field (associated with an ipsilateral increase in alpha amplitude), the involuntary capture of attention by a sound only involves a facilitation of visual processing associated with enhanced contralateral alpha blocking. This hypothesis is fully consistent with findings from slow potential (LDAP and ACOP) recordings reviewed above. 
A ERP waveform showing the ACOP

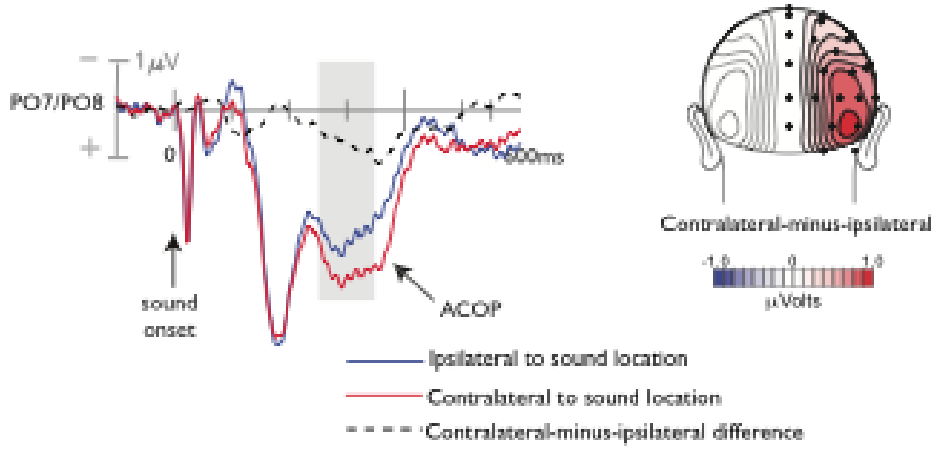

B Time-frequency plot showing the alpha blocking
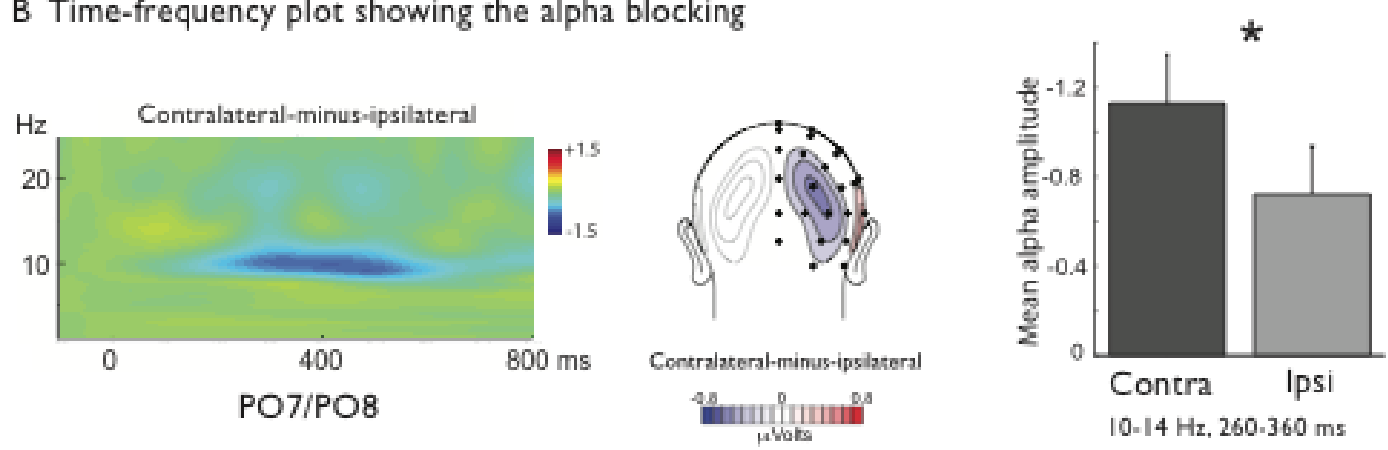

Figure 5. ACOP component in the time-domain ERP elicited by lateralized, task-irrelevant sounds is paralleled by alpha rhythm desynchronization in the frequency domain. A. Grand average ERP waveforms elicited at occipital scalp sites show greater positivity over the contralateral hemisphere over the interval 200-500 ms (ACOP). Scalp topography of the ACOP mapped as the contralateral minus ipsilateral positivity over $250-350 \mathrm{~ms}$ shows a sharp occipital focus. B. Time-frequency plot of neural activity following the sound shows a decrease (blocking) of alpha band power that was greater in the hemisphere contralateral to the sound. This plot shows the contralateral minus ipsilateral power changes quantified by wavelets. Scalp topography of the lateralized alpha amplitude change is very similar to that of the ACOP. Bar graph indicates that alpha rhythm was blocked (relative to pre-stimulus baseline) in both hemispheres, more so in the hemisphere contralateral to the sound. 
Störmer et al. obtained further evidence linking the ACOP with alpha desynchronization by sorting individual trials (lateralized sound presentations) into those with higher and those with lower ACOPs for each subject. It was found that trials with a larger ACOP were associated with significantly larger decreases in contralateral alphaband power. There was also a between-subjects correlation, such that observers who showed larger ACOPs to the lateralized sounds also showed stronger contralateral alphaband desynchronization. These correlations plus their temporal and anatomical correspondences suggest that the ACOP and alpha blocking elicited by an irrelevant but salient sound may arise from a common physiological mechanism. Such a mechanism has been proposed by Mazaheri \& Jensen (2008), who demonstrated how slow ERP components (such as the ACOP) could be generated by asymmetrical modulations of the oscillatory alpha rhythm. Their idea is that modulations of the alpha rhythm instigated by a cognitive process like the orienting of attention, might have a stronger effect on the peaks of the rhythm than the troughs (or vice versa). If such an asymmetry occurred systematically on multiple trials the net result would be a preferential accumulation of peaks of one polarity or the other in the averaged waveform, and if those peaks were not time-locked to the eliciting event, the result would be a slow potential in the average ERP waveform (Van Dijk et al., 2010). If this account is correct, the ACOP may simply be a manifestation of an asymmetrical blocking (desynchronization) of the alpha rhythm asymmetrical in the sense of being greater in the hemisphere contralateral to the auditory stimulus and in the sense of peaks and troughs being differentially modulated.

There is an interesting contrast between the results of the electrophysiological studies of cross-modal orienting reviewed here and those of hemodynamic neuroimaging 
studies (e.g., Laurienti et al. 2002), which have found that the presentation of a stimulus in one modality (auditory or visual) produces a deactivation of the sensory cortex of the other modality. In contrast, the sound-evoked electrophysiological responses in the visual cortex (ACOP and alpha blocking) appear to be facilitatory to visual perception rather than inhibitory. The ACOP amplitude was found to correlate positively with perceived visual contrast (McDonald et al. 2013b) and with visual discrimination accuracy (Feng et al. 2014), while alpha blocking has been shown in numerous studies (reviewed above) to be associated with improved visual perception and has thus been linked to facilitation. It would be very interesting to conduct a combined fMRI/EEG study using a cross-modal orienting paradigm like those reviewed here in an effort to resolve the apparent discrepancy between the deactivation seen with fMRI and the facilitation seen with EEG/ERP recordings. Some possible reasons for the discrepancy might be differences in experimental paradigms (e.g., the type of attention that is engaged), differences in the temporal sensitivity of the two classes of measures, or a surprising dissociation between net neural activity and improved processing.

\section{Functional Selectivity of Cross-Modal Orienting}

The cross-modal interactions reviewed in this article present an interesting case of functional selectivity in that they show both selective and non-selective properties. The facilitatory effect of a peripheral sound on the perceptual processing of a subsequent visual stimulus is clearly selective with respect to spatial position; that is, the effect is greatest when the auditory and visual stimuli are spatially coincident. The neural substrates of this cross-modal facilitation also show functionally selectivity in that they 


\section{Cross-modal orienting of visual attention}

include a contralateral pattern of slow potential shift and alpha desynchronization in the ventral visual cortex. If, as we propose, this cross-modal facilitation is a consequence of the orienting of visual attention to the sound's location, then the sound-evoked neural modulations in the visual cortex are dependent upon the activation of a broader network of brain areas that underlies attentional orienting. This attention-orienting network is also functionally specialized and includes multiple cortical and subcortical areas (McDonald et al. 2012).

While the attention-orienting network is functionally specialized for stimulus location, it is not specialized for stimulus modality. It is well established that a lateralized cue in one modality (auditory, visual or tactile) facilitates the processing of a subsequent target stimulus in either the same or a different modality (Spence \& Driver 2004; McDonald et al. 2012). This lack of modality specificity holds true both for the voluntary orienting of attention and the involuntary orienting by spatially non-predictive cues. The studies reviewed here provide another example of the modality non-specificity of involuntary orienting, and the observed neural correlates provide information about the time course and neural substrates of these cross-modal influences. Further work is needed to determine whether all of these cross-modal cueing effects are mediated by a unitary orienting system that receives spatially coded input from all modalities or whether more specialized direct connections between the different modalities are also involved (Driver \& Noesselt 2008).

\section{Summary and Conclusions}


The studies reviewed here point to the conclusion that a salient sound attracts visual attention to its location even when it provides no information about where a subsequent visual target may occur, or even when no visual stimuli are presented at all. This capture of attention was found to affect the perception of a visual target at the sound's location in three different ways: (1) improving its detection and discrimination; (2) speeding the timing of its perceptual awareness; and (3) enhancing its perceived contrast. In traditional terminology such a spatially non-predictive sound would be regarded as an "exogenous" cue that attracted attention involuntarily, as opposed to an "endogenous" (or symbolic) cue indicating the location where attention was to be directed voluntarily. This involuntary orienting of attention to the salient sound was found to be associated with a slow positive ERP arising from the visual cortex (the ACOP) that was larger in the hemisphere contralateral to the sound's location. If the time course of the ACOP (ca. 200-500 ms after the cue) is an accurate reflection of the timing of facilitated processing in the visual cortex due to attention, this suggests that exogenous cross-modal cueing would only be effective at relatively short SOAs in this time range. This is consistent with effects of exogenous visual cues on behavioral performance, which are usually observed at shorter cue-target SOAs (100-200ms), but not at longer cue-target intervals (Nakayama \& Mackeben, 1989). Future studies should examine the temporal correspondence between these behavioral benefits and the ACOP more systematically.

Remarkably, the ACOP could be elicited not only by a spatially non-predictive sound preceding a visual target but also by a completely irrelevant sound presented in a randomized sequence with auditory targets. These findings led McDonald et al. (2013b) 
to characterize the orienting of attention to salient sounds as "automatic", in that the sound's relevance did not seem to matter. It remains to be seen, however, whether this involuntary orienting indexed by the ACOP can be modified by some manipulations of attention or other cognitive processes. As far as we know, there are no cortical ERPs with latencies longer than $100 \mathrm{~ms}$ that have proved to be totally automatic in the sense of being elicited identically under all possible task conditions and brain states. However, it should be noted that the initial evoked response of the primary visual cortex (the "C1" component at 50-80 ms) appears to be impervious to manipulations of attention (Ding et al. 2014) and thus may qualify for full automaticity.

A new development in this line of research was the observation that the ACOP is paralleled by (and perhaps equivalent to) a lateralized desynchronization (blocking) of the occipital alpha rhythm. It has long been known that lateralized modulations of the alpha rhythm accompany the voluntarily allocation of attention (see references above), but it now appears that similar modulations also accompany the involuntary capture of attention (see also Mlynarski et al., 2014). Further studies are needed to determine whether the slow potential shift appearing as the ACOP has an influence on visual processing over and above that associated with the alpha modulation, or whether they are two manifestations of the same process. Further research is also needed to determine the extent to which spatial allocations of attention are associated with alpha blocking (facilitating the processing of attended-location stimuli) as opposed to alpha enhancement (inhibiting the processing of unattended locations) and how such modulations actually improve or impair the quality of visual information passing through the cortical pathways. 
Cross-modal orienting of visual attention

\section{Acknowledgments}

This research was supported by grants from the National Science Foundation (BCS1029084) and the National Institute of Mental Health (1P50MH86385) to S.A.H., and from the Natural Sciences and Engineering Research Council of Canada and the Canada Foundation for Innovation to J.J.M., and by a Marie Curie fellowship (EU Grant PIOFGA-2012-329920) to V.S.S. The authors thank Matthew M. Marlow for technical assistance. We are grateful to the editor and two referees for helpful suggestions that have improved this article. 


\section{References}

Baart, M., Stekelenburg, J. J., \& Vroomen, J. (2014). Electrophysiological evidence for speech-specific audiovisual integration. Neuropsychologia, 53, 115-121.

Bonath, B., Noesselt, T., Martinez, A., Mishra, J., Schwiecker, K., Heinze, H. J., \& Hillyard, S. A. (2007). Neural basis of the ventriloquist illusion. Current Biology, 17(19), 1697-1703.

Calvert, G., Spence, C., \& Stein, B. E. (Eds.). (2004). The handbook of multisensory processes. MIT press.

Carrasco, M., Ling, S., \& Read, S. (2004). Attention alters appearance. Nature Neuroscience, 7(3), 308-313.

Capilla, A., Schoffelen, J. M., Paterson, G., Thut, G., \& Gross, J. (2014). Dissociated $\alpha-$ band modulations in the dorsal and ventral visual pathways in visuospatial attention and perception. Cerebral Cortex, 24(2), 550-561.

Ding, Y., Martinez, A., Qu, Z. \& Hillyard, S.A. (2014). The earliest stages of visual cortical processing are not modified by attentional load. Human Brain Mapping, 35, 3008-3024.

Driver, J., \& Noesselt, T. (2008). Multisensory interplay reveals crossmodal influences on 'sensory-specific' brain regions, neural responses, and judgments. Neuron, 57(1), 1123.

Driver, J., \& Spence, C. (2004). Crossmodal spatial attention: Evidence from human performance. In C. Spence \& J. Driver (eds.), Crossmodal Space and Crossmodal Attention (pp. 179-220). Oxford University Press.

Dufour, A.(1999). Importance of attentional mechanisms in audiovisual links. Experimental Brain Research, 126(2), 215-222.

Eimer, M., \& Schröger, E. (1998). ERP effects of intermodal attention and cross-modal links in spatial attention. Psychophysiology, 35(03), 313-327.

Ergenoglu, T., Demiralp, T., Bayraktaroglu, Z., Ergen, M., Beydagi, H., \& Uresin, Y. (2004). Alpha rhythm of the EEG modulates visual detection performance in humans. Cognitive Brain Research, 20(3), 376-383.

Feng, W., Störmer, V. S., Martinez, A., McDonald, J. J., \& Hillyard, S. A. (2014). Sounds activate visual cortex and improve visual discrimination. The Journal of Neuroscience, 34(29), 9817-9824. 
Foxe, J. J., \& Snyder, A. C. (2011). The role of alpha-band brain oscillations as a sensory suppression mechanism during selective attention. Frontiers in Psychology, 2,154. doi: 10.3389/fpsyg.2011.00154

Frassinetti, F., Bolognini, N., \& Làdavas, E. (2002). Enhancement of visual perception by crossmodal visuo-auditory interaction. Experimental Brain Research, 147(3), 332-343.

Ganesh, A. C., Berthommier, F., Vilain, C., Sato, M., \& Schwartz, J. L. (2014). A possible neurophysiological correlate of audiovisual binding and unbinding in speech perception. Frontiers in Psychology, 5, 1340. doi:10.3389/fpsyg.2014.01340.

Green, J. J. \& McDonald, J. J. (2010). The role of temporal predictability in the anticipatory biasing of sensory cortex during visuospatial shifts of attention. Psychophysiology, 47, 1057-1065.

Green, J.J., Teder-Sälejärvi, W.A., \& McDonald, J.J. (2005). Control mechanisms mediating shifts of attention in auditory and visual space: a spatio-temporal ERP analysis. Exp Brain Res. 166(3-4), 358-69.

Händel, B. F., Haarmeier, T., \& Jensen, O. (2011). Alpha oscillations correlate with the successful inhibition of unattended stimuli. Journal of Cognitive Neuroscience, 23(9), 2494-2502.

Hanslmayr, S., Aslan, A., Staudigl, T., Klimesch, W., Herrmann, C. S., \& Bäuml, K. H. (2007). Prestimulus oscillations predict visual perception performance between and within subjects. Neuroimage, 37(4), 1465-1473.

Hanslmayr, S., Gross, J., Klimesch, W., \& Shapiro, K. L. (2011). The role of alpha oscillations in temporal attention. Brain Research Reviews, 67(1), 331-343.

Harter MR, Miller SL, Price NJ, Lalonde ME, Keyes AL. (1989). Neural processes involved in directing attention. J Cogn Neurosci, 1(3), 223-37.

Heinze, H.J., Mangun, G.R. , Burchert, W. , Hinrichs, H., Scholz, M. , Münte, T.F. , Gos, AScherg, . M. , Johannes, S. , Hundeshagen, H. , Gazzaniga, M.S. \& Hillyard, S.A. (1994). Combined spatial and temporal imaging of brain activity during visual selective attention in humans. Nature, 372, 543-546.

Hikosaka, O., Miyauchi, S., \& Shimojo, S. (1996). Orienting of spatial attention-its reflexive, compensatory, and voluntary mechanisms. Cognitive Brain Research, 5(1-2), $1-9$.

Hillyard, S.A., Simpson, G.V., Woods, D.L., Van Voorhis, S. \& Münte, T. (1984). Event-related brain potentials and selective attention to different modalities. In F. Reinoso-Suarez \& C. Ajmone-Marsan (eds.). Cortical Integration (pp. 395-414). New York: Raven Press. 
Hopf, J.M., \& Mangun, G.R. (2000). Shifting visual attention in space: an electrophysiological analysis using high spatial resolution mapping. Clin Neurophysiol., 111(7), 1241-57.

Hopfinger, J.B., Luck, S.J. \& Hillyard, S.A. (2004). Selective attention: Electrophysiological and neuromagnetic studies. In: M.S. Gazzaniga (Ed.). The Cognitive Neurosciences III, MIT Press, pp. 561-574.

James,W. (1890). The Principles of Psychology. Henry Holt, New York, NY.

Jensen, O., \& Mazaheri, A. (2010). Shaping functional architecture by oscillatory alpha activity: Gating by inhibition. Frontiers in Human Neuroscience, 4, 186.

doi:10.3389/fnhum.2010.00186

Kelly, S. P., Lalor, E. C., Reilly, R. B., \& Foxe, J. J. (2006). Increases in alpha oscillatory power reflect an active retinotopic mechanism for distracter suppression during sustained visuospatial attention. Journal of Neurophysiology, 95(6), 3844-3851.

Klimesch, W. (2012). Alpha-band oscillations, attention, and controlled access to stored information. Trends in Cognitive Science, 16(12), 606-617.

Laurienti, P.J., Burdette, J.H., Wallace, M.T., Yen, Y.F., Field, A.S., \& Stein, B.E. (2002). Deactivation of sensory-specific cortex by cross-modal stimuli. J Cogn Neurosci., 14(3), 420-9.

Luck, S.J. \& Kappenman, E.S. (eds.) (2011). The Oxford Handbook of Event-Related Potential Components. Oxford University Press.

Marshall, T. R., O'Shea, J., Jensen, O., \& Bergmann, T. O. (2015). Frontal eye fields control attentional modulation of alpha and gamma oscillations in contralateral occipitoparietal cortex. The Journal of Neuroscience, 35(4), 1638-1647.

Mazaheri, A., \& Jensen, O. (2008). Asymmetric amplitude modulations of brain oscillations generate slow evoked responses. The Journal of Neuroscience, 28(31), 77817787.

Mazaheri, A., van Schouwenburg, M. R., Dimitrijevic, A., Denys, D., Cools, R., \& Jensen, O. (2014). Region-specific modulations in oscillatory alpha activity serve to facilitate processing in the visual and auditory modalities. Neuroimage, 87, 356-362.

McDonald, J.J., Green, J.J., Sto $\square$ rmer, V.S., \& Hillyard, S.A. (2012). Cross-modal spatial cueing of attention influences visual perception. In M.M. Murray \& M.T. Wallace (eds.), Frontiers in the Neural Basis of Multisensory Processes (pp. 509-528), CRC Press. 
McDonald, J. J., Störmer, V. S., Martinez, A., Feng, W., \& Hillyard, S. A. (2013b).

Salient sounds activate human visual cortex automatically. The Journal of

Neuroscience, 33(21), 9194-9201.

McDonald, J. J., Teder-Sälejärvi, W. A., \& Hillyard, S. A. (2000). Involuntary orienting to sound improves visual perception. Nature, 407(6806), 906-908.

McDonald, J. J., Teder-Sälejärvi, W. A., Di Russo, F., \& Hillyard, S. A. (2003). Neural substrates of perceptual enhancement by cross-modal spatial attention.Journal of Cognitive Neuroscience, 15(1), 10-19.

McDonald, J. J., Teder-Sälejärvi, W. A., Di Russo, F., \& Hillyard, S. A. (2005). Neural basis of auditory-induced shifts in visual time-order perception. Nature Neuroscience, 8(9), 1197-1202.

McDonald, J.J., \& Green, J.J. (2008). Isolating event-related potential components associated with voluntary control of visuo-spatial attention. Brain Res, 1227, 96-109.

McDonald, J. J., Whitman, J., Sto $\square$ rmer, V. S., \& Hillyard, S. A. (2013a). Involuntary cross-modal spatial attention influences visual perception. In G.R. Mangun, (ed.), Cognitive electrophysiology of attention: Signals of the mind (pp. 82-95). Elsevier Inc.

Mishra, J., Martinez, A., Sejnowski, T. J., \& Hillyard, S. A. (2007). Early cross-modal interactions in auditory and visual cortex underlie a sound-induced visual illusion. The Journal of Neuroscience, 27(15), 4120-4131.

Murray, M. M., \& Wallace, M. T. (Eds.). (2012). The neural bases of multisensory processes. CRC Press.

Mlynarski, W., Freigang, C., Bennemann, J., Stöhr, M., \& Rübsamen, R. (2014). Position of acoustic stimulus modulates visual $\alpha$ activity. NeuroReport, 25(11), 833-837.

Nakayama, K., \& Mackeben, M. (1989). Sustained and transient components of focal visual attention. Vision research, 29(11), 1631-1647.

Payne, L., Guillory, S., \& Sekuler, R. (2013). Attention-modulated alpha-band oscillations protect against intrusion of irrelevant information. Journal of Cognitive Neuroscience, 25(9), 1463-1476.

Prinzmetal, W., Long, V., \& Leonhardt, J. (2008). Involuntary attention and brightness contrast. Perception \& Psychophysics, 70(7), 1139-1150.

Santangelo, V., \& Spence, C. (2008). Crossmodal attentional capture in an unspeeded simultaneity judgement task. Visual Cognition,16, 155-165. 
Schneider, K. A., \& Bavelier, D. (2003). Components of visual prior entry. Cognitive Psychology, 47(4), 333-366.

Schneider, K. A., \& Komlos, M. (2008). Attention biases decisions but does not alter appearance. Journal of Vision, 8(15), 3, 1:10.

Shams, L., Kamitani, Y., \& Shimojo, S. (2000). Illusions: What you see is what you hear. Nature, 408(6814), 788-788.

Shimojo, S., Miyauchi, S., \& Hikosaka, O. (1997). Visual motion sensation yielded by non-visually driven attention. Vision Research, 37(12), 1575-1580.

Shore, D. I., Spence, C., \& Klein, R. M. (2001). Visual prior entry. Psychological Science, 12(3), 205-212.

Spence, C. \& Driver, J. (Eds.) (2004) Crossmodal space and crossmodal attention. Oxford University Press.

Störmer, V. S., Feng, W., Martinez, A., McDonald, J. J., \& Hillyard, S. A. (submitted). Salient sounds elicit slow potential shifts paralleled by alpha rhythm desynchronization in visual cortex.

Störmer, V.S., Green, J.J., \& McDonald, J.J. (2009). Tracking the voluntary control of auditory spatial attention with event-related brain potentials. Psychophysiology, 46, 357366.

Störmer, V. S., McDonald, J. J., \& Hillyard, S. A. (2009). Cross-modal cueing of attention alters appearance and early cortical processing of visual stimuli. Proceedings of the National Academy of Sciences, 106(52), 22456-22461.

Teder-Sälejärvi, W. A., Münte, T. F., Sperlich, F. J., \& Hillyard, S. A. (1999). Intramodal and cross-modal spatial attention to auditory and visual stimuli. An event-related brain potential study. Cognitive Brain Research, 8(3), 327-343.

Thut, G., Miniussi, C., \& Gross, J. (2012). The functional importance of rhythmic activity in the brain. Current Biology, 22(16), R658-R663.

Thut, G., Nietzel, A., Brandt, S. A., \& Pascual-Leone, A. (2006). $\alpha$-Band electroencephalographic activity over occipital cortex indexes visuospatial attention bias and predicts visual target detection. The Journal of Neuroscience, 26(37), 9494-9502.

van Dijk, H., Schoffelen, J. M., Oostenveld, R., \& Jensen, O. (2008). Prestimulus oscillatory activity in the alpha band predicts visual discrimination ability. The Journal of Neuroscience, 28(8), 1816-1823. 
van Dijk, H., van der Werf, J., Mazaheri, A., Medendorp, W. P., \& Jensen, O. (2010). Modulations in oscillatory activity with amplitude asymmetry can produce cognitively relevant event-related responses. Proceedings of the National Academy of Sciences, 107(2), 900-905.

van Velzen, J., Forster, B., Eimer, M. (2002). Temporal dynamics of lateralized ERP components elicited during endogenous attentional shifts to relevant tactile events. Psychophysiology. 39(6), 874-8.

Worden, M. S., Foxe, J. J., Wang, N., \& Simpson, G. V. (2000). Anticipatory biasing of visuospatial attention indexed by retinotopically specific-band electroencephalography increases over occipital cortex. Journal of Neuroscience, 20(6), 1-6. 